\title{
THE HILBERT POLYNOMIAL OF A SYMBOLIC SQUARE
}

\author{
KALOYAN SLAVOV
}

\begin{abstract}
Let $k$ be an algebraically closed field, and let $C \subset \mathbb{P}_{k}^{n}$ be a reduced closed subscheme with ideal sheaf $\mathcal{I}$. Let $\mathcal{I}^{<2>}$ be the second symbolic power of $\mathcal{I}$. When $C$ is an integral curve, we compute the Hilbert polynomial of $\mathcal{O}_{\mathbb{P}^{n}} / \mathcal{I}^{<2>}$ in terms of invariants of $C$.
\end{abstract}

\section{INTRODUCTION}

Fix an algebraically closed field $k$. Let $i: C \hookrightarrow \mathbb{P}_{k}^{n}$ be a reduced closed subscheme, and let $\mathcal{I}$ be its ideal sheaf. Let $S=k\left[x_{0}, \ldots, x_{n}\right]$ with the usual grading.

Following Lazarsfeld (see p. 177 in [11] or p. 164 in [12]), define the second symbolic power $\mathcal{I}^{<2>}$ of $\mathcal{I}$ as the ideal sheaf consisting of germs of functions that vanish to order at least 2 at every point of $C$. When $C$ is integral, Corollary 1 in 44 implies that for any affine open $U=\operatorname{Spec} R \subset \mathbb{P}^{n}$, the ideal $\Gamma\left(U, \mathcal{I}^{<2>}\right) \subset R$ coincides with the classical second symbolic power of $\Gamma(U, \mathcal{I})$, as traditionally defined in commutative algebra - i.e., it is the primary component of the ordinary second power of $\Gamma(U, \mathcal{I})$. See also the discussion in Section 3.9 in [3]. When $C$ is a local complete intersection, we have $\mathcal{I}^{<2>}=\mathcal{I}^{2}$; however, this can fail for other subschemes $C$ (see Example 2.2 in Section 2).

The study of the symbolic powers of various closed subschemes has received recent interest; see, e.g., [1], 2], [6], 77. See [9] for another instance when the second symbolic power of the ideal of a subscheme is of interest, in connection with the birational projection of a variety onto a hypersurface.

The motivation to investigate the Hilbert polynomial of $\mathcal{O}_{\mathbb{P}^{n}} / \mathcal{I}^{<2>}$ when $\operatorname{dim} C \geq 1$ came up as we were working on the problem of identifying the dimension of the moduli space of hypersurfaces $V(F)$ of degree $l$ in $\mathbb{P}^{n}$ whose singular locus has dimension at least $b$, for a given

1991 Mathematics Subject Classification. Primary 14N99; Secondary 14H50, 14J17.

Key words and phrases. symbolic power, Kähler differentials, singular locus, Hilbert polynomial, curve, hypersurface. 
$b \geq 1$; see [14]. Namely, for a fixed reduced $C \hookrightarrow \mathbb{P}^{n}$ with ideal sheaf $\mathcal{I}$, Lazarsfeld's definition of $\mathcal{I}^{<2>}$ directly implies that for $F \in S_{l}$, we have $C \subset V(F)_{\text {sing }}$ if and only if $F \in \Gamma\left(\mathbb{P}^{n}, \mathcal{I}^{<2>}(l)\right)$. In [14], we give an upper bound for $\operatorname{dim} \Gamma\left(\mathbb{P}^{n}, \mathcal{I}^{<2>}(l)\right)$ that is valid for all $l$.

Our first task now is to describe the sheaf $\mathcal{I}^{<2>}$ in a more explicit manner. Namely, in Section 2, we recall that $\mathcal{I}^{<2>}$ fits into the exact sequence

$$
0 \rightarrow \mathcal{I}^{<2>} \rightarrow \mathcal{I} \rightarrow \Omega_{\mathbb{P} n} / \mathcal{I} \Omega_{\mathbb{P} n} \rightarrow i_{*} \Omega_{C} \rightarrow 0
$$

In Section 3 , we turn to the natural question of computing the Hilbert polynomial of $\mathcal{O}_{\mathbb{P}^{n}} / \mathcal{I}^{<2>}$. Let $P_{C}(l)$ be the Hilbert polynomial of $C$. We prove the following

Proposition 1.1. The Hilbert polynomial of $\mathcal{O}_{\mathbb{P}^{n}} / \mathcal{I}^{<2>}$ is given by

$$
\operatorname{dim}_{k} \Gamma\left(\mathbb{P}^{n},\left(\mathcal{O}_{\mathbb{P}^{n}} / \mathcal{I}^{<2>}\right)(l)\right)=(n+1) P_{C}(l-1)-\operatorname{dim}_{k} \Gamma\left(C, \Omega_{C}(l)\right)
$$

for $l \gg 0$.

The goal of Section 4 is to refine Proposition 1.1 in the case when $C$ is an integral curve. For this, we need to investigate the term $\Gamma\left(C, \Omega_{C}(l)\right)$ in more detail.

Let $C$ be an integral curve over $k$, and let $p: \widetilde{C} \rightarrow C$ be its normalization. Let $\Psi: \Omega_{C} \rightarrow p_{*} \Omega_{\widetilde{C}}$ be the canonical map, and let $\mathcal{R}_{1}, \mathcal{R}_{2}$ denote its kernel and cokernel:

$$
0 \rightarrow \mathcal{R}_{1} \rightarrow \Omega_{C} \stackrel{\Psi}{\rightarrow} p_{*} \Omega_{\widetilde{C}} \rightarrow \mathcal{R}_{2} \rightarrow 0 .
$$

Since $p$ is an isomorphism over a dense open subset of $C$, so is $\Psi$, and hence $\mathcal{R}_{1}$ and $\mathcal{R}_{2}$ have finite support, contained in $C_{\text {sing. In particular, }}$ $\mathcal{R}_{1}$ and $\mathcal{R}_{2}$ are torsion sheaves. Note that the torsion subsheaf of $\Omega_{C}$ is contained in $\mathcal{R}_{1}$, since $p_{*} \Omega_{\widetilde{C}}$ is locally free, as a $p_{*} \mathcal{O}_{\widetilde{C}}$-module; therefore, $\mathcal{R}_{1}$ is the torsion subsheaf of $\Omega_{C}$. See the proof of Proposition $2.2 \mathrm{in}$ [5] for its rich history. For each $P \in C_{\text {sing }}$, the stalks $\left(\mathcal{R}_{1}\right)_{P}$ and $\left(\mathcal{R}_{2}\right)_{P}$ are finite-dimensional $k$-vector spaces, so we can define the invariant

$$
\mu(C):=\sum_{P \in C_{\text {sing }}}\left(\operatorname{dim}_{k}\left(\mathcal{R}_{1}\right)_{P}-\operatorname{dim}_{k}\left(\mathcal{R}_{2}\right)_{P}\right)
$$

For an integral curve $i: C \hookrightarrow \mathbb{P}^{n}$ with ideal sheaf $\mathcal{I}$ and saturated ideal $I$, we let $d$ be its degree and $p_{a}$ be its arithmetic genus, so for large $l$, we have

$$
P_{C}(l)=\operatorname{dim}_{k}(S / I)_{l}=d l+1-p_{a} .
$$

Notation as in [5], let $p_{g}$ denote the geometric genus of $C$, i.e., the genus of $\widetilde{C}$. We prove 
Proposition 1.2. For $l \gg 0$,

$\operatorname{dim}_{k} \Gamma\left(\mathbb{P}^{n},\left(\mathcal{O}_{\mathbb{P}^{n}} / \mathcal{I}^{<2>}\right)(l)\right)=n d l+1+(n+1)\left(1-d-p_{a}\right)-p_{g}-\mu(C)$.

When $C$ is an integral curve that is a local complete intersection, Proposition 2.2 in [5] implies that $\chi\left(\Omega_{C}\right)=\chi\left(\omega_{C}\right)$, where $\omega_{C}$ is the dualizing sheaf of $C$. As a consequence,

$$
\mu(C)=\chi\left(\Omega_{C}\right)-\chi\left(\Omega_{\widetilde{C}}\right)=\chi\left(\omega_{C}\right)-\chi\left(\Omega_{\widetilde{C}}\right)=p_{a}-p_{g} .
$$

We recover this result in the particular case when $C$ is a plane curve, by an elementary argument. Namely, we compute the codimension of $\Gamma\left(\mathbb{P}^{n}, \mathcal{I}^{<2>}(l)\right)$ in $S_{l}$ directly and compare with the formula in Proposition 1.2. As an easy corollary, we deduce:

Corollary 1.3. For an integral plane curve $C \hookrightarrow \mathbb{P}^{2} \subset \mathbb{P}^{n}$, the Hilbert polynomial of the sheaf $\Omega_{C}$ of Kähler differentials is

$$
\chi\left(\Omega_{C}(l)\right)=d l+p_{a}-1 .
$$

Note that $C$ is not required to be smooth.

Finally, in Section 5, we compute explicitly the invariant $\mu(C)$ in the following

Example 1.4. Consider the ideal

$$
I=\left(y^{2}-x z, y z-x^{3}, z^{2}-x^{2} y\right) \subset A=k[x, y, z],
$$

and let $C^{0}=V(I) \subset \mathbb{A}^{3}$; this is the curve parametrized by

$$
x=t^{3}, y=t^{4}, z=t^{5} .
$$

The projective closure $C \subset \mathbb{P}^{3}$ of $C^{0}$ has $d=5, p_{a}=2$, and $p_{g}=0$. The unique singular point of $C$ is $(0,0,0) \in C^{0}$.

In Section 5, we compute that $\mu(C)=3$, so the Hilbert polynomial of $\mathcal{O}_{\mathbb{P}^{3}} / \mathcal{I}^{<2>}$ is $15 l-26$.

\section{AN EXACT SEQUENCE FOR $\mathcal{I}^{<2>}$}

We investigate the sheaf $\mathcal{I}^{<2>}$. Proposition 2.1 below is a particular case of Exercise 8 on p. 83 in [10]. We include its proof for completeness.

Proposition 2.1. a) Let $I \subset A=k\left[x_{1}, \ldots, x_{n}\right]$ be a radical ideal. Then

$$
I^{<2>}=\operatorname{Ker}\left(I \rightarrow \Omega_{A} / I \Omega_{A}\right) .
$$


b) Let $i: C \hookrightarrow \mathbb{P}^{n}$ be a reduced closed subscheme with ideal sheaf $\mathcal{I}$. There is an exact sequence

$$
0 \rightarrow \mathcal{I}^{<2>} \rightarrow \mathcal{I} \rightarrow \Omega_{\mathbb{P} n} / \mathcal{I} \Omega_{\mathbb{P}^{n}} \rightarrow i_{*} \Omega_{C} \rightarrow 0 .
$$

Proof. It suffices to prove part a). Suppose that $f \in I$ satisfies $f \in \mathfrak{m}^{2}$ for all $\mathfrak{m} \supset I$. We claim that $d f \in I \Omega_{A / k}$, where $d: A \rightarrow \Omega_{A / k}$ is the canonical derivation. (In this way, we linearize the a-priori inconvenient condition that $f \in \mathfrak{m}^{2}$. We know that for each maximal $\mathfrak{m} \supset I$, we have $\Omega_{A / k} / \mathfrak{m} \Omega_{A / k}=\Omega_{A / k} \otimes_{A} A / \mathfrak{m} \simeq \mathfrak{m} / \mathfrak{m}^{2}$ as $A / \mathfrak{m}$-vector spaces, and

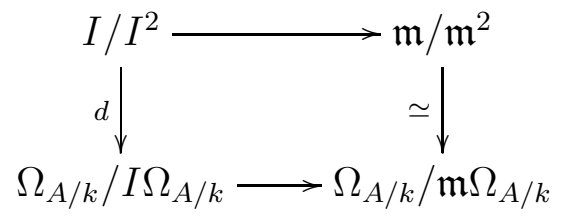

commutes, so the condition $f \in \mathfrak{m}^{2}$ is equivalent to $d f \in \mathfrak{m} \Omega_{A / k}$.

Since $\Omega_{A / k}$ is a free $A$-module, we conclude that

$$
d f \in \bigcap_{\mathfrak{m} \supset I}\left(\mathfrak{m} \Omega_{A / k}\right)=\left(\bigcap_{\mathfrak{m} \supset I} \mathfrak{m}\right) \Omega_{A / k}=I \Omega_{A / k} .
$$

The converse is obvious from the commutative diagram above.

Remark 2.2. Notation as in Example 1.4, consider

$$
f=x y\left(y^{2}-x z\right)-x^{2}\left(y z-x^{3}\right)+z\left(z^{2}-x^{2} y\right) \in I .
$$

An easy check shows that $d f=0$ in $\Omega_{A} / I \Omega_{A}$, so $f \in I^{<2>}$. However, every element of $I^{2}$ contains only monomials of degree 4 or larger, and since $f$ contains the monomial $z^{3}$ of degree 3 , we deduce that $f \notin I^{2}$. Thus, $I^{2}$ is strictly smaller than $I^{<2>}$ in this example.

Corollary 2.3. Suppose that $C \subset \mathbb{P}^{n}$ is a reduced closed subscheme, which is a local complete intersection. Let $\mathcal{I}$ be the ideal sheaf of $C$. Then for $F \in S_{l}$ we have $C \subset V(F)_{\text {sing }}$ if and only if $F \in \Gamma\left(\mathbb{P}^{n}, \mathcal{I}^{2}(l)\right)$.

Proof. The condition $C \subset V(F)_{\text {sing }}$ is equivalent to $F \in \Gamma\left(\mathcal{I}^{<2>}(l)\right)$. The inclusion $\mathcal{I}^{2} \subset \mathcal{I}^{<2>}$ is an equality if $C$ is a local complete intersection, since in this case, the map $i^{*} \mathcal{I} \rightarrow i^{*} \Omega_{\mathbb{P} n}$ is injective (e.g. Exercise 16.17 in [3]).

\section{The Hilbert polynomial of $\mathcal{O}_{\mathbb{P}^{n}} / \mathcal{I}^{<2>}$}

Let $i: C \hookrightarrow \mathbb{P}^{n}$ be a reduced closed subscheme with ideal sheaf $\mathcal{I}$. 
Proof of Proposition 1.1. Define $\mathcal{H} \in \operatorname{Coh}(C)$ by the exactness of

$$
0 \rightarrow \mathcal{H} \rightarrow i^{*} \Omega_{\mathbb{P}^{n}} \rightarrow \Omega_{C} \rightarrow 0,
$$

so (2) yields a short exact sequence

$$
0 \rightarrow \mathcal{I}^{<2>} \rightarrow \mathcal{I} \rightarrow i_{*} \mathcal{H} \rightarrow 0 .
$$

From the short exact sequence

$$
0 \rightarrow i_{*} \mathcal{H} \rightarrow \mathcal{O}_{\mathbb{P}^{n}} / \mathcal{I}^{<2>} \rightarrow \mathcal{O}_{\mathbb{P}^{n}} / \mathcal{I} \rightarrow 0
$$

we obtain a short exact sequence

$$
0 \rightarrow \Gamma\left(\mathbb{P}^{n}, i_{*} \mathcal{H}(l)\right) \rightarrow \Gamma\left(\mathbb{P}^{n},\left(\mathcal{O}_{\mathbb{P}^{n}} / \mathcal{I}^{<2>}\right)(l)\right) \rightarrow \Gamma\left(\mathbb{P}^{n},\left(\mathcal{O}_{\mathbb{P}^{n}} / \mathcal{I}\right)(l)\right) \rightarrow 0
$$

for large $l$. The last term has dimension $P_{C}(l)$ for large $l$, so it suffices to compute the dimension of the first term.

The short exact sequence

$$
0 \rightarrow i_{*} \mathcal{H} \rightarrow \Omega_{\mathbb{P}^{n}} / \mathcal{I} \Omega_{\mathbb{P}^{n}} \rightarrow i_{*} \Omega_{C} \rightarrow 0
$$

gives rise to a short exact sequence

$$
0 \rightarrow \Gamma\left(\mathbb{P}^{n}, i_{*} \mathcal{H}(l)\right) \rightarrow \Gamma\left(\mathbb{P}^{n},\left(\Omega_{\mathbb{P}^{n}} / \mathcal{I} \Omega_{\mathbb{P}^{n}}\right)(l)\right) \rightarrow \Gamma\left(C, \Omega_{C}(l)\right) \rightarrow 0
$$

for $l$ sufficiently large.

Finally, we have to compute $\operatorname{dim}_{k} \Gamma\left(\mathbb{P}^{n},\left(\Omega_{\mathbb{P}^{n}} / \mathcal{I} \Omega_{\mathbb{P}^{n}}\right)(l)\right)$ for large $l$. Recall (e.g. Theorem II.8.13 in [8]) the short exact sequence

$$
0 \rightarrow \Omega_{\mathbb{P}^{n}} \rightarrow \mathcal{O}_{\mathbb{P}^{n}}(-1)^{\oplus(n+1)} \rightarrow \mathcal{O}_{\mathbb{P}^{n}} \rightarrow 0 .
$$

Since $\mathcal{O}_{\mathbb{P}^{n}}$ is locally free, applying $-\otimes_{\mathcal{O}^{n} n} \mathcal{O}_{\mathbb{P}^{n}} / \mathcal{I}$ to this short exact sequence yields a short exact sequence

$$
0 \rightarrow \frac{\Omega_{\mathbb{P}^{n}}}{\mathcal{I} \Omega_{\mathbb{P}^{n}}} \rightarrow\left(\frac{\mathcal{O}_{\mathbb{P}^{n}}(-1)}{\mathcal{I} \mathcal{O}_{\mathbb{P}^{n}}(-1)}\right)^{\oplus(n+1)} \rightarrow \frac{\mathcal{O}_{\mathbb{P}^{n}}}{\mathcal{I}} \rightarrow 0
$$

The sequence

$$
0 \rightarrow \Gamma\left(\frac{\Omega_{\mathbb{P}^{n}}}{\mathcal{I} \Omega_{\mathbb{P}^{n}}}(l)\right) \rightarrow \Gamma\left(\frac{\mathcal{O}_{\mathbb{P}^{n}}(-1)}{\mathcal{I} \mathcal{O}_{\mathbb{P}^{n}}(-1)}(l)\right)^{\oplus(n+1)} \rightarrow \Gamma\left(\frac{\mathcal{O}_{\mathbb{P}^{n}}}{\mathcal{I}}(l)\right) \rightarrow 0
$$

is exact for large $l$. For large $l$, the third term has dimension $P_{C}(l)$ as before.

We are left to compute $\operatorname{dim}_{k} \Gamma\left(\mathbb{P}^{n},(\mathcal{O}(-1) / \mathcal{I} \mathcal{O}(-1))(l)\right)$ for large $l$. Notice that $\mathcal{I O}(-1) \simeq \mathcal{I}(-1)$ and that for large $l$,

$$
\Gamma\left(\mathbb{P}^{n},(\mathcal{O}(-1) / \mathcal{I}(-1))(l)\right)=\Gamma\left(\mathbb{P}^{n},\left(\mathcal{O}_{\mathbb{P}^{n}} / \mathcal{I}\right)(l-1)\right)
$$

has dimension $P_{C}(l-1)$.

Going back through the exact sequences, we complete the calculation. 
For example, if $C \simeq \mathbb{P}^{r}$ is an $r$-dimensional projective linear subspace, we know the Hilbert polynomial $P_{C}(l)$, and we can easily determine the dimensions $\operatorname{dim}_{k} \Gamma\left(\mathbb{P}^{r}, \Omega_{\mathbb{P}^{r}}(l)\right)$ for large $l$, by using the Euler sequence (41) for $\Omega_{\mathbb{P}^{r}}$. This computes the Hilbert polynomial of $\mathcal{O}_{\mathbb{P}^{n}} / \mathcal{I}^{<2>}$, and hence the Hilbert polynomial of $\mathcal{I}^{<2>}$. This approach generalizes to the case when $C$ is a disjoint union of linear subspaces, hence we obtain a weak version of Lemma 2.3 in [2].

As an easy special case, let $C=\left\{P_{1}, \ldots, P_{d}\right\}$ be a finite set of points (with reduced induced structure). Since $\Omega_{C}=0$, we have $\mathcal{H} \simeq i^{*} \Omega_{\mathbb{P}^{n}}$, and hence an exact sequence

$$
0 \rightarrow \Omega_{\mathbb{P}^{n}} / \mathcal{I} \Omega_{\mathbb{P}^{n}} \rightarrow \mathcal{O}_{\mathbb{P}^{n}} / \mathcal{I}^{<2>} \rightarrow \mathcal{O}_{\mathbb{P}^{n}} / \mathcal{I} \rightarrow 0 .
$$

However, $\Omega_{\mathbb{P}^{n}} / \mathcal{I} \Omega_{\mathbb{P}^{n}}=\Omega_{\mathbb{P}^{n}} \otimes_{\mathcal{O}_{\mathbb{P}} n}\left(\mathcal{O}_{\mathbb{P}^{n}} / \mathcal{I}\right)$ has zero-dimensional support (the same is true for all of its twists by $\mathcal{O}_{\mathbb{P}^{n}}(l)$ ), hence

$$
H^{1}\left(\left(\Omega_{\mathbb{P} n} / \mathcal{I} \Omega_{\mathbb{P}^{n}}\right)(l)\right)=0 .
$$

Thus, for all $l$, we have an exact sequence

$$
0 \rightarrow \Gamma\left(\left(\Omega_{\mathbb{P}^{n}} / \mathcal{I} \Omega_{\mathbb{P}^{n}}\right)(l)\right) \rightarrow \Gamma\left(\left(\mathcal{O}_{\mathbb{P}^{n}} / \mathcal{I}^{<2>}\right)(l)\right) \rightarrow \Gamma\left(\left(\mathcal{O}_{\mathbb{P}^{n}} / \mathcal{I}\right)(l)\right) \rightarrow 0 .
$$

This vanishing of $H^{1}\left(\mathbb{P}^{n},\left(\Omega_{\mathbb{P}^{n}} / \mathcal{I} \Omega_{\mathbb{P}^{n}}\right)(l)\right)$ also implies that the sequence (5) is exact for all $l$. Therefore,

$$
\operatorname{dim}_{k} \Gamma\left(\left(\mathcal{O}_{\mathbb{P}^{n}} / \mathcal{I}^{<2>}\right)(l)\right)=(n+1) \operatorname{dim}_{k} \Gamma\left(\left(\mathcal{O}_{\mathbb{P}^{n}} / \mathcal{I}\right)(l-1)\right)=(n+1) d
$$

for all $l$. See [7] for a discussion of the more subtle question of the Hilbert function of the saturated ideal of $\mathcal{I}^{2}$.

\section{The Case When $C$ is a CuRve}

Lemma 4.1. Let $i: C \hookrightarrow \mathbb{P}^{n}$ be an integral curve. Notation as in Section 1, we have

$$
\operatorname{dim}_{k} \Gamma\left(C, \Omega_{C}(l)\right)=d l+p_{g}-1+\mu(C) \quad \text { for } l \gg 0 .
$$

Proof. For large $l$, the sequence

(6)

$$
0 \rightarrow \Gamma\left(C, \mathcal{R}_{1}(l)\right) \rightarrow \Gamma\left(C, \Omega_{C}(l)\right) \rightarrow \Gamma\left(C,\left(p_{*} \Omega_{\widetilde{C}}\right)(l)\right) \rightarrow \Gamma\left(C, \mathcal{R}_{2}(l)\right) \rightarrow 0
$$

is exact.

Note that

$$
\Gamma\left(C, \mathcal{R}_{1}(l)\right) \simeq \Gamma\left(C, \mathcal{R}_{1}\right)=\bigoplus_{P \in C_{\text {sing }}}\left(\mathcal{R}_{1}\right)_{P}
$$

and similarly for $\mathcal{R}_{2}$. 
Now, we look at the term $\Gamma\left(C,\left(p_{*} \Omega_{\widetilde{C}}\right)(l)\right)$. By the projection formula, we know

$$
\left(p_{*} \Omega_{\widetilde{C}}\right)(l) \simeq p_{*}\left(\Omega_{\widetilde{C}} \otimes_{\mathcal{O}_{\widetilde{C}}} p^{*} \mathcal{O}_{C}(l)\right) .
$$

Since $C$ has degree $d, p^{*} \mathcal{O}_{C}(l)$ is a line bundle on $\widetilde{C}$ of degree $d l$ (e.g. Proposition 3.8 on p. 276 in [13]). By the Riemann-Roch theorem applied to $\widetilde{C}$, it follows that for large $l$,

$$
\operatorname{dim}_{k} \Gamma\left(\widetilde{C}, \Omega_{\widetilde{C}} \otimes p^{*} \mathcal{O} C(l)\right)=d l+p_{g}-1 .
$$

Taking the alternating sum of dimensions in (6) completes the proof.

Proof of Proposition 1.2. Combine Proposition 1.1, Lemma 4.1, and (1).

Let $I=\left(f, x_{b+2}, \ldots, x_{n}\right) \subset S$, where $f \in k\left[x_{0}, \ldots, x_{b+1}\right]_{d}-\{0\}$ (for us, the important case will be $b=1$ ). Consider the (surjective) composition

$\Phi: k\left[x_{0}, \ldots, x_{b+1}\right]_{l} \oplus\left(\bigoplus_{i=b+2}^{n} k\left[x_{0}, \ldots, x_{b+1}\right]_{l-1} x_{i}\right) \hookrightarrow S_{l} \rightarrow S_{l} /\left(I^{2} \cap S_{l}\right)$.

Lemma 4.2. We have that

$$
\operatorname{ker}(\Phi)=\left\{P+\sum_{i=b+2}^{n} P_{i} x_{i}: f^{2}|P, f| P_{i} \text { for } i=b+2, \ldots, n\right\} .
$$

For $l \geq 2 d$, the codimension of $I_{l}^{2}=I^{2} \cap S_{l}$ in $S_{l}$ equals $\beta_{d}(l)$, where

$$
\begin{aligned}
\beta_{d}(l)=\left(\begin{array}{c}
l+b+1 \\
b+1
\end{array}\right)- & \left(\begin{array}{c}
l-2 d+b+1 \\
b+1
\end{array}\right)+ \\
& (n-b-1)\left(\left(\begin{array}{l}
l+b \\
b+1
\end{array}\right)-\left(\begin{array}{c}
l-d+b \\
b+1
\end{array}\right)\right) .
\end{aligned}
$$

Proof. If $P+\sum P_{i} x_{i} \in I^{2}$, just expand it as a polynomial in $x_{b+2}, \ldots, x_{n}$. The second part is an immediate consequence.

Corollary 4.3. For an integral plane curve $C$, we have $\mu(C)=p_{a}-p_{g}$.

Proof. We compute the codimension of $\Gamma\left(\mathbb{P}^{n}, \mathcal{I}^{<2>}(l)\right)$ in $S_{l}$ for large $l$ in two different ways. On the one hand, it is given by the formula in Proposition 1.2. On the other hand, since $C$ is a (local) complete intersection, we know that $\mathcal{I}^{<2>}=\mathcal{I}^{2}$, and since the ideal $I^{2}$ is saturated, we have, explicitly, $\Gamma\left(\mathbb{P}^{n}, \mathcal{I}^{<2>}(l)\right)=I_{l}^{2}$. Thus, the codimension we are computing equals the codimension of $I_{l}^{2}$ in $S_{l}$, which we computed as 
$\beta_{d}(l)$ in Lemma $4.2($ take $b=1)$. We equate the two linear polynomials in $l$ and compare their constant coefficients. Recall that $p_{a}=\frac{(d-1)(d-2)}{2}$ to obtain the desired conclusion.

Remark 4.4. The conclusion of Corollary 4.3 can fail for a general integral curve $C \subset \mathbb{P}^{n}$. For instance, if $C$ is the curve defined in Example 1.4, then $\mu(C)=3$ by Lemma 5.1 in Section 5, while $p_{a}-p_{g}=2$.

Proof of Corollary 1.3. Combine Lemma 4.1 and Corollary 4.3.

\section{An EXPLicit EXAMPLE}

Assume that $\operatorname{char}(k) \neq 2,3,5$. Notation as in Example 1.4, let $B=$ $A / I$, and note that $\Omega_{B}=(B d x \oplus B d y \oplus B d z) /\left(\eta_{1}, \eta_{2}, \eta_{3}\right)$, where

$$
\begin{aligned}
& \eta_{1}=-z d x+2 y d y-x d z, \\
& \eta_{2}=-3 x^{2} d x+z d y+y d z, \\
& \eta_{3}=2 x y d x+x^{2} d y-2 z d z .
\end{aligned}
$$

Consider the map $\Psi: \Omega_{B} \rightarrow k[t] d t$ induced by $x \mapsto t^{3}, y \mapsto t^{4}, z \mapsto t^{5}$. The cokernel of $\Psi$ has dimension 2 over $k$; now we investigate $\operatorname{ker}(\Psi)$ :

Lemma 5.1. We have $\operatorname{dim}_{k} \operatorname{ker}(\Psi)=5$.

Proof. First note that any element $b \in B$ can be written uniquely as

$$
b=\alpha(x)+\beta(x) y+\gamma(x) z,
$$

where $\alpha, \beta, \gamma \in k[x]$. Indeed, existence follows by using the relations in $B$, and for uniqueness, suppose that an element $\alpha(x)+\beta(x) y+\gamma(x) z$ of $A$ (with $\alpha, \beta, \gamma \in k[x]$ ) belongs to $I$. Since $I$ contains only monomials of degree 2 or larger, it follows that $\alpha=x \alpha_{1}, \beta=x \beta_{1}, \gamma=x \gamma_{1}$. But then, since $I$ is prime, $\alpha_{1}+\beta_{1} y+\gamma_{1} z$ must be in $I$. Continuing the process, we deduce that $\alpha, \beta, \gamma \in\left(x^{n}\right)$ for all $n$, hence $\alpha=\beta=\gamma=0$.

Consider a differential

$w=\left(P_{1}+Q_{1} y+R_{1} z\right) d x+\left(P_{2}+Q_{2} y+R_{2} z\right) d y+\left(P_{3}+Q_{3} y+R_{3} z\right) d z$ in $\Omega_{B}$, where all $P_{i}, Q_{i}, R_{i}$ are in $k[x]$. Split the image of $w$ under the composition $\Omega_{B} \stackrel{\Psi}{\rightarrow} k[t] d t \stackrel{\simeq}{\rightarrow} k[t]$ as a sum of three polynomials according to the residues modulo 3 of the exponents of the monomials that they contain. We deduce that $w$ belongs to $\operatorname{ker}(\Psi)$ if and only if

$$
\begin{gathered}
3 P_{1}(x)+4 x^{2} R_{2}(x)+5 x^{2} Q_{3}(x)=0 \\
3 x Q_{1}(x)+4 P_{2}(x)+5 x^{2} R_{3}(x)=0 \\
3 x R_{1}(x)+4 x Q_{2}(x)+5 P_{3}(x)=0,
\end{gathered}
$$


in $k[x]$, i.e.,

$$
\begin{aligned}
& P_{1}(x)=-\frac{1}{3}\left(4 x^{2} R_{2}(x)+5 x^{2} Q_{3}(x)\right) \\
& P_{2}(x)=-\frac{1}{4}\left(3 x Q_{1}(x)+5 x^{2} R_{3}(x)\right) \\
& P_{3}(x)=-\frac{1}{5}\left(3 x R_{1}(x)+4 x Q_{2}(x)\right) .
\end{aligned}
$$

Going back to the expression of $w$, it follows that $\Psi(w)=0$ if and only if $w$ is a $k[x]$-linear combination of the following 6 differentials in $\Omega_{B}$ :

$$
\begin{gathered}
w_{1}:=5 z d x-3 x d z, w_{2}:=-5 x^{2} d x+3 y d z, w_{3}:=4 y d x-3 x d y, \\
w_{4}:=-4 x^{2} d x+3 z d y, w_{5}:=-5 x^{2} d y+4 z d z, w_{6}:=5 y d y-4 x d z .
\end{gathered}
$$

However, since $w_{4}=-w_{2}, w_{5}=x w_{3}$ and $w_{6}=\frac{w_{1}}{2}$, it follows that $\operatorname{ker}(\Psi)$ is the $k[x]$-submodule of $\Omega_{B}$ generated by $w_{1}, w_{2}, w_{3}$. Next, it is easy to see that $x^{2} w_{1}=0, x w_{2}=0, x^{2} w_{3}=0$ in $\Omega_{B}$. Therefore, the 5 differentials $w_{1}, x w_{1}, w_{2}, w_{3}, x w_{3}$ span $\operatorname{ker}(\Psi)$ as a $k$-vector space.

Suppose that $a_{1}, \ldots, a_{5} \in k$ give a linear dependence relation among these 5 differentials. Working in $B d x \oplus B d y \oplus B d z$, this means that there exist $\alpha_{i}, \beta_{i}, \gamma_{i} \in k[x]$, for $i=1,2,3$, such that

$$
a_{1} w_{1}+a_{2} x w_{1}+a_{3} w_{2}+a_{4} w_{3}+a_{5} x w_{3}=\sum_{i=1}^{3}\left(\alpha_{i}+\beta_{i} y+\gamma_{i} z\right) \eta_{i}
$$

(by abuse of notation, the obvious lift of $w_{i}$ is still denoted by $w_{i}$ ). Comparing the coefficients of $d y, d z$, and then in turn using uniqueness of the decomposition (8) , we obtain the following equalities in $k[x]$ :

$$
\begin{aligned}
-3\left(a_{4}+a_{5} x\right) x & =2 x^{3} \gamma_{1}+x^{3} \beta_{2}+x^{2} \alpha_{3} \\
0 & =2 \alpha_{1}+x^{2} \gamma_{2}+x^{2} \beta_{3} \\
0 & =\alpha_{2}+2 x \beta_{1}+x^{2} \gamma_{3} \\
-3\left(a_{1}+a_{2} x\right) x & =-x \alpha_{1}+x^{3} \gamma_{2}-2 x^{3} \beta_{3} \\
3 a_{3} & =-x \beta_{1}+\alpha_{2}-2 x^{2} \gamma_{3} \\
0 & =-2 \alpha_{3}-x \gamma_{1}+x \beta_{2} .
\end{aligned}
$$

By (10), we have $\alpha_{1} \in\left(x^{2}\right)$, hence (12) implies that $a_{1}=a_{2}=0$. Next, by (11), we have $\alpha_{2} \in(x)$, so (13) yields $a_{3}=0$. Finally, (14) implies that $\alpha_{3} \in(x)$, hence (9) gives $a_{4}=a_{5}=0$. 


\section{FURTHER STUDY}

We can list a number of questions related to the current study. For example, given a closed subscheme $C \hookrightarrow \mathbb{P}^{n}$ (not necessarily reduced), it would be interesting to describe the space of all hypersurfaces $F \in S_{l}$ for which there is a scheme-theoretic inclusion

$$
C \hookrightarrow V\left(F, \frac{\partial F}{\partial x_{0}}, \ldots, \frac{\partial F}{\partial x_{n}}\right):=\operatorname{Proj}\left(S /\left(F, \frac{\partial F}{\partial x_{i}}\right)\right) .
$$

Also, given an integral curve $C \subset \mathbb{P}^{n}$, it would be interesting to investigate the Hilbert polynomials of higher symbolic powers of $\mathcal{I}$. For example, if we mimic the discussion in Section 2, we find that the third symbolic power $\mathcal{I}^{<3>}$ fits into an exact sequence

$$
0 \rightarrow \mathcal{I}^{<3>} \rightarrow \mathcal{I}^{<2>} \rightarrow \Omega_{\mathbb{P}^{n}} / \mathcal{I}^{<2>} \Omega_{\mathbb{P}^{n}} \rightarrow j_{*} \Omega_{D} \rightarrow 0,
$$

where $j: D \hookrightarrow \mathbb{P}^{n}$ is the closed subscheme whose ideal sheaf is $\mathcal{I}^{<2>}$ (of course, $C \simeq D_{\text {red }}$ ). However, the Hilbert polynomial $\chi\left(\Omega_{D}(l)\right.$ ) is more difficult to analyze.

Finally, when $C \subset \mathbb{P}^{n}$ is a curve, the question of the Hilbert function of $I^{<2>}$ (the saturated ideal of $\mathcal{I}^{2}$ ) appears naturally. As suggested, for example, in [1] and [7], this question will be interesting and non-trivial, since it is such already when $C$ is a finite set of points.

\section{ACKNOWLEDGMENTS}

The problem that we address in this article came up naturally as I was working on my doctoral thesis at MIT under the direction of Bjorn Poonen. I am grateful to Prof. Poonen for all of his dedication and substantial help throughout the entire process. I also thank an anonymous referee for some helpful comments and suggestions. I thank Steven Kleiman for a number of references and thorough comments. Finally, I thank Martin Kreuzer for some additional references.

\section{REFERENCES}

[1] C. Ciliberto, R. Miranda, Linear Systems of Plane Curves with Base Points of Equal Multiplicity, Trans. Amer. Math. Soc. 352 (2000), no. 9, 4037-4050.

[2] M. Dumnicki, B. Harbourne, T. Szemberg, H. Tutaj-Gasinska, Linear subspaces, symbolic powers and Nagata type conjectures, arXiv:1207.1159.

[3] D. Eisenbud, Commutative algebra with a view toward algebraic geometry, Springer-Verlag, New York, 1995, Graduate Texts in Mathematics, No. 150.

[4] D. Eisenbud, M. Hochster, A Nullstellensatz with Nilpotents and Zariski's Main Lemma on Holomorphic Functions, Journal of Algebra, 58 (1979), 157161. 
[5] E. Esteves, S. Kleiman, Bounds on leaves of one-dimensional foliations, Dedicated to the 50-th anniversary of IMPA. Bull. Braz. Math. Soc. (N.S.) 34 (2003), $145-169$.

[6] A. V. Geramita, B. Harbourne, J. Migliore, Star configurations in $\mathbb{P}^{n}$, arXiv: 1203.5685

[7] B. Harbourne, Generators for Symbolic Powers of Ideals Defining General Points of $\mathbb{P}^{2}$, arXiv:alg-geom/9509003

[8] R. Hartshorne, Algebraic Geometry, Springer-Verlag, New York, 1977, Graduate Texts in Mathematics, No. 52.

[9] S. Kleiman, B. Ulrich, Gorenstein algebras, symmetric matrices, self-linked ideals, and symbolic powers, Trans. Amer. Math. Soc. 349 (1997), 4973-5000.

[10] E. Kunz, Algebraic Differential Calculus (Preliminary version, last change: March, 2002), Chapter 1.5, available at http://www-nw.uni-regensburg. de/ kue22107/index.htm.

[11] R. K. Lazarsfeld, Positivity in algebraic geometry, Vol. I, Springer, 2000, A Series of Modern Surveys in Mathematics, No. 48.

[12] R. K. Lazarsfeld, Positivity in algebraic geometry, Vol. II, Springer, 2000, A Series of Modern Surveys in Mathematics, No. 49.

[13] Q. Liu, Algebraic Geometry and Arithmetic Curves, Oxford University Press, 2002.

[14] K. Slavov, The moduli space of hypersurfaces whose singular locus has high dimension, Math. Zeitschrift, 2014, DOI: 10.1007/s00209-014-1360-0; arXiv:1208.1118 